\title{
ПРОБЛЕМЫ МЕТОДОЛОГИИ
}

Удк 373.51

\author{
С. Н. Дегтярев
}

\section{КАЧЕСТВЕННЫЕ И КОЛИЧЕСТВЕННЫЕ МЕТОДЫ \\ В ПЕДАГОГИЧЕСКОМ ИССЛЕДОВАНИИ КРЕАТИВНЫХ СПОСОБНОСТЕЙ УЧАЩИХСЯ}

Аннотаиия. В статье раскрываются функции качественных и количественных методов научного исследования. Показаны области их применения, достоинства и ограничения. Доказывается, что, несмотря на заметно возросшие возможности математического аппарата, компьютеризацию обработки формальных данных, в педагогике качественные методы остаются приоритетными, поскольку дают возможность понять суть педагогического явления, его движущие силы и отношения. Приводится убедительный пример сравнительного эксперимента, демонстрирующего неуниверсальность количественных методов.

В реальном исследовании и те, и другие методы используются как отдельные процедуры. Однако существуют современные исследовательские методики, в которых сочетаются или интегрируются оба подхода. Автор показывает возможность такого сочетания в методике семантического диффреренциала, которая была использована при изучении креативности (творческих способностей) учащихся на базе гимназии Тюменского государственного университета.

Материалы статьи будут интересны всем, кто занимается научными изысканиями в сфере педагогики и образования, в частности тем, кто изучает методы исследования творческих способностей учащихся.

Ключевые слова: качественные методы, количественные методы, креативность, статистическая закономерность, функции методов исследования.

Abstract. The paper investigates the qualitative and quantitative research methods, their application fields, advantages and limitations. The author argues that, in spite of the high capabilities of modern mathematical apparatus and information technologies, the qualitative research methods still retain their priority in pedagogy. The examples of comparative experimental analysis are given demonstrating the lack of universality typical of the quantitative methods.

Both methods are generally applied as the separate procedures; however, there are some modern research methods integrating the above ap- 
proaches. The paper demonstrates such integration by means of the Semantic Differential Method implemented in the students' creativity studies in the Grammar school of Tyumen State University. The research materials can be of interest to specialists in education and pedagogy exploring the students' creative abilities.

Keywords: qualitative methods, quantitative methods, creativity, statistical conformities, functions of research methods.

Аюбое исследование, в том числе и педагогическое, невозможно полноценно выполнить, опираясь только на качественные или только на количественные методы. При всей мощи современных комичественных методов (кластерного анализа, корреляционного, факторного анализа и др.), выросших возможностях математического аппарата, компьютеризации обработки формальных данных, исследователь, опираясь только на них, часто не может проникнуть в суть изучаемых явлений, понять причинно-следственные связи и генетическую основу развития (изменения) изучаемого объекта. Использование только качественных методов анализа не позволяет выйти на уровень статистически значимых обобщений, описать поведение не отдельных, разрозненных компонентов, а системы в цемом, в ее динамике, с прогнозированием, определением возможного уровня состояния и развития в будущем.

Выбор и обоснование комплекса методов, наиболее адекватно и полно позволяющих изучить объект исследования, является методологической проблемой для автора научной работы. От ее успешного решения зависят степень достижения исследовательских целей и достоверность полученных результатов. С развитием исследовательского аппарата, увеличением количества методов и методик данная проблема становится все более актуальной. Верный выбор можно сделать мишь хорошо понимая функции и соотношение различных методов, область их применения и ограничения.

К функииям качественных методов относятся:

- выявление значимых соииально-педагогических фактов, законов, поиск новых идей, подходов через наблюдение, интерпретаиию, моделирование, прогнозирование, содержательный анализ полученной информации: качественные методы могут придавать значимость единичным фактам, за которыми могут скрываться серьезные проблемы, требующие своего изучения и разрешения. Несмотря на то, что математический инструментарий не межит в ос- 
нове качественных методов, последние могут быть эффективными и в работе с количественными данными, представленными, например и задачах, в проблемных ситуациях. Исходную цифровую, графическую, условно-знаковую информацию можно преобразовать в комплексную модель текста [3, с. 111], отражающую взаимосвязи и отношения (прямые и косвенные, явные и скрытые) этой информации;

- формирование иелостного образа изучаемого объекта: если с помощью количественных методов изучаются мишь определенные составцяющие, стороны объекта, которые могут быть измерены, то качественные методы на основе интерпретации и анализа не поддающихся количественному измерению важных составляющих объекта (процесса) позволяют создать о нем целостное представление;

- компенсаиия определенных, слабо разработанных аспектов психолого-педагогической теории путем эмпирического взаимодействия с объектом исследования: речь идет о поисковой работе, немыслимой без широкого применения качественных методов. Эмпирическое взаимодействие способствует глубокому "погружению" в изучаемый объект, накоплению сведений, фактов, их сопоставмению, обобщению, выдвижению идей преобразования, гипотез и дальнейшей разработке теории объекта (процесса);

- обеспечение гибкости исследовательского проиесса: количественные методы в силу их специфики, стандартизации процедур могут применяться только в определенных условиях формализации объекта, а качественные используются в "непроходимых" для количественных методов областях и обеспечивают комплексное изучение объекта. Например, количественные методы будут малоэффективны при изучении истинных мотивов поведения ребенка, причин его переживаний, стрессов - здесь нужны именно качественные методы. По мнению Д. Равена, таковыми могут быть наблюдение за учеником в процессе выполнения им заданий; фиксация многочисленных и взаимозаменяемых компетентностей, которые проявцяют учащиеся при выполнении этих заданий; использование описательного подхода при заполнении протоколов наблюдения вместо фиксации результатов в бамлах [5, с. 20].

Нельзя при помощи количественных методов описать и творческий процесс обучения, вдохновение, творческий порыв ребен- 
ка. Способность к творчеству - чрезвычайно важная характери-

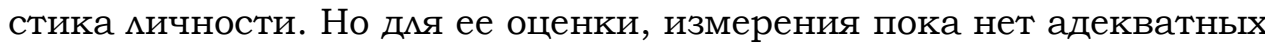
математических модемей и инструментов расчета. Учитывая индивидуальный характер проявления творческих качеств мичности, нужно не измерять, а образно представить данный процесс, увидеть его детали, "прикоснуться" к неуловимым эмоциональным и мысмительным движениям ученика;

- формирование новых понятий (системы понятий): это важный "доколичественный" этап в познавательном процессе, можно сказать, стадия открытия новых значений. Выйти на новые понятия или уточнить уже широко используемые понятия и категории возможно посредством содержательного анализа, через выявмение новых сторон изучаемого объекта. Количественные же методы могут быть использованы дия проверки, подтверждения предположений в отношении установменных значений.

К основным функииям количественных методов могут быть отнесены:

- проверка статистических гипотез, которая дает возможность сделать выводы о достоверности полученных результатов, т. е. перенести их на всю генеральную совокупность (при выполнении выборочного исследования), на другие ситуации, объекты или процессы;

- количественное описание изучаемого объекта, определение его количественных характеристик, например показателей центральной тенденции (среднего значения переменной, моды, медианы, среднего квадратичного отклонения) или характеристик корреляции (согласования) и др. Изучаемым объектом может быть и текст. Так, содержание какого-цибо учебного курса может быть представлено в виде некоторой математической модеми на основе геометрической топологии через использование фракталов и мультифракталов, позволяющих описать интегративные связи структуры учебного материала [1, с. 7];

- построение интерполяиионных и экстраполяиионных математических моделей, которые позволяют по ограниченному набору данных получить информацию о поведении изучаемого объекта (его характеристиках) внутри измеряемого интервала (интерполяция) и вне его (экстраполяция);

- выявление направленности изменения изучаемого объекта (проиесса), тендениий его развития (прогресс, регресс) на основе 
математических модемей через изучение (измерение) его составмяющих (их динамики), изменения связей между ними;

- обнаружение научных фактов путем анализа больших объемов статистического материала, выявление закономерностей, значимых изменений в размичных системах образования.

Разделить методы исследования на качественные и количественные можно по ключевым формулировкам вопросов. Количественный подход ориентирует исследователя на поиск ответов: "сколько?", "во сколько раз?", "как часто?" - т. е. на сбор исчисляемых данных, исходя из которых определяются рейтинги, коэффициенты, процентные доли, строятся графики, диаграммы и т. п.

Качественные методы дают ответы на вопросы: "почему?", "зачем?", "из-за чего?", "каким образом?" - и позволяют произвести более гибкий анализ причинно-следственных связей, установить структуру и компоненты изучаемого объекта (процесса). Качественный подход опирается на интуицию, мнения, суждения отдельных субъектов, которые в некоторых случаях могут быть более значимыми, чем статистические данные.

K количественным методам относят методы статистической обработки (описательная статистика, сравнение выборок и рядов показателей, анализ временных рядов, расчетная оценка надежности методик), корреляционный анализ, факторный анализ, дискриминантный анализ, кластерный анализ, моделирование, тестирование, анкетирование. К качественным - беседу, интервью, наблюдение, контент-анализ, изучение продуктов деятельности, экспертную оценку, фокус-группу, интерпретацию, прогнозирование, Аичностный дифференциал, биографический метод, метод интелмектуального диалога, метафорическое моделирование, этимологический анализ.

Сторонники количественного подхода, считающие главным его преимуществом достоверность и доказательность, критикуют качественные методы за субъективизм и низкую достоверность результатов. Однако известный американский ученый-методомог Д. Кэмпбемц настаивал, что они обладают внутренними механизмами валидизаиии (валидный - адекватный целям, замыслу), обеспечивающими соответствие и прямую связь применяемых методов и фиксируемых экспериментальных эффектов. Это дает основание считать, что с помощью данных методов может быть получена достоверная инфор- 
мация. Д. Кэмпбем отмечал, что в реальной практике может происходить даже перепроверка количественных результатов качественными методами [4, с. 327]. Там, где качественные оценки приходят в противоречие с количественными результатами, последние следует брать под сомнение, пока не будут найдены причины расхождения. Неадекватность количественных данных часто обнаруживается кишь благодаря качественному знанию.

Кроме того, следует обратить внимание еще на одно уязвимое место количественных методов. Закономерности, полученные на основе обработки статистического материала (количественных данных), могут описывать поведение (изменение, динамику) всей системы (совокупности эмементов, измерение которых и даяо необходимый количественный материал), но они не могут быть применены к описанию поведения конкретного элемента (если это выборка - то отдельного индивида), даже в том случае, когда достоверность выводов подтверждена разцичными статистическими критериями. В качестве подтверждения этой мысли приведем краткое описание одного эксперимента, выполненного нами с целью выяснения влияния предварительной активации ранее сформированных установок на процесс принятия индивидом интуитивного решения (отметим попутно, что, с нашей точки зрения, механизмы интуитивного и креативного решений являются во многом идентичными).

Суть эксперимента, в котором приняли участие 107 человек (педагоги и старшеклассники), состояна в том, что двум группам испытуемых предъявцялась фотография одного и того же не выражающего никаких эмоций человека, но на разном фоне. В одном случае (фото 1) это ухоженный дом, сад из цветущих растений, во втором (фото 2) - заброшенный квартал, серое полуразрушенное здание, замусоренная территория. Участникам опыта необходимо бымо, исходя из внутренних ощущений, описать характер человека, выбрав из предложенного перечня определенные ка-

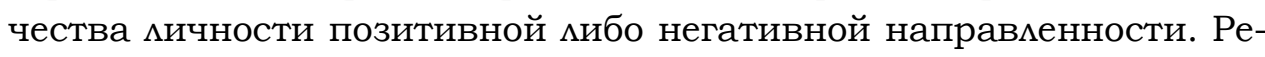
зультаты эксперимента представцены в таблице.

Итак, количественные данные приводят нас к однозначному заключению: актуализированная (в том числе и подсознательно) информация повлияла на характер интуитивного решения. "Позитивный" фон предопределил доминирование положительных качеств в первой группе, "негативный" - отрицательных качеств во 
второй. Вывод относительно предварительной активизации внутренних установок очевиден, даже нет необходимости в специальной статистической обработке данных.

Выбор оценочных суждений в зависимости от характера актуализированной информации

\begin{tabular}{|l|c|c|c|c|}
\hline \multirow{2}{*}{$\begin{array}{c}\text { Группы } \\
\text { испытуемых }\end{array}$} & $\begin{array}{c}\text { Положительные } \\
\text { качества }\end{array}$ & $\begin{array}{c}\text { Отрицательные } \\
\text { качества }\end{array}$ & $\begin{array}{c}\text { Положительные } \\
\text { качества }\end{array}$ & $\begin{array}{c}\text { Отрицательные } \\
\text { качества }\end{array}$ \\
\hline Педагоги & 56 & 18 & 20 & 37 \\
\hline Учащиеся & 144 & 91 & 53 & 125 \\
\hline
\end{tabular}

Однако и среди взрослых, и среди учащихся нашиись мюди, ответы которых были противоположны большинству (на "негативном" фоне испытуемые выбирали положительные характеристики, на "позитивном" - отрицательные). Таких испытуемых оказалось немного, но благодаря этим фактам можно утверждать следующее: полученная статистическая закономерность, определяющая общую тенденцию оценок ("негативный" фон - негативные характеристики, "позитивный" фон - позитивные характеристики), не может быть применена к каждому конкретному индивиду (испытуемому). Всегда существует вероятность противоположного ответа, обратной реакции, исключения. Это общее свойство всех статистических законов. Причем оно характерно не только дмя гуманитарных наук. В физике тоже имеются подобные примеры: так, закон радиоактивного распада описывает закономерность распада огромного количества ядер, но абсолютно не применим к описанию распада одного ядра.

В реальном исследовании качественные и количественные методы используются как отдельные процедуры. Есть современные исследовательские методики, в которых эти подходы сочетаются или интегрируются. Ярким примером их необходимого и результативного сочетания является методика семантического дифференциала, которая может быть использована для изучения креативности (творческих способностей) учащихся.

Базой данной методики являются такие количественные методы обработки информации, как корреляционный, факторный и кластерный виды статистического анализа, позволяющие опреде- 
мять уровень взаимосвязи изучаемых факторов и сгруппировать информацию (выполнить ее кластеризацию). Однако сбор информации происходит с помощью качественных методов, например через организацию работы фокус-группы, в которой отбираются дескрипторы (признаки, описывающие объект изучения), значимые с точки зрения участников этой группы. Полученные с помощью факторного анализа результаты могут быть интерпретированы только качественным способом. Выделенные факторы, включающие наиболее значимые связи, могут быть осмыслены, оценены, интерпретированы благодаря знаниям исследователя, его опыту и интуиции.

Креативность - сложное качество мичности, напрямую не измеряемое. Дця нее нет эталона, единицы измерения, нет интервальных шкал. Но оценить ее можно по косвенным признакам, способами реконструирования индивидуальных и групповых семантических пространств и измерения так называемых коннотативных значений, т. е. субъективных состояний, следующих за восприятием символараздражителя. Методика семантического дифференциала является комбинацией процедур шкалирования и метода контролируемых ассоциаций, который приемлем для косвенной оценки развития креативности Аичности исходя из связи уровня развития творческих способностей человека и его представлений о креативной мичности и факторах креативной среды. Косвенные оценки в некоторых случаях явцяются единственным источником информации об изучаемом объекте. Это касается величин (явлений), слабо поддающихся формализации, дмя которых невозможно сконструировать измерительную шкалу, подобрать единицу измерения и соответствующий ей эталон (точную меру, служащую для воспроизведения, хранения и передачи информации о какой-либо величине). Ведь, действительно, трудно представить эталоны мотивации, активности, пассивности, креативности и т. д. [2, с. 20]. Поэтому и необходимы косвенные показатели, суть применения которых заключается в установлении связи изучаемых характеристик объекта, не поддающихся формальному подсчету, с параметрами, измерение которых возможно с удовметворяющей исследователя точностью.

Методику семантического дифференциала мы использовали при изучении различий в субъективных представлениях о чертах креативной мичности и факторах креативной среды у учащихся, поступивших в гимназию Тюменского государственного универси- 
тета (10-е классы) и выпускников гимназии (11-е классы). Всего в исследовании приняли участие 88 человек.

На первом этапе эксперимента с помощью работы фокусгруппы (15 учащихся) и с помощью экспертов (6 учителей и психомогов) был составлен перечень креативных характеристик мичности и факторов креативной среды.

На втором этапе проводилось анкетирование учащихся, результаты которого обрабатывались с помощью статистических методов (компьютерная программа "Statistica"), на основании чего были выделены черты креативной мичности и факторы креативной среды, соответствующие взглядам учащихся 10-х и 11-х классов.

После осуществлялась качественная оценка обнаруженных размичий с выдемением их возможных причин.

Мы исходили из тех соображений, что субъективные представления меняются в сознании ученика под воздействием образовательной среды и ее творческого потенциала. Фиксация подобных изменений позволяет, с нашей точки зрения, судить (конечно, косвенно) о креативном потенциале среды и о ее качестве. Таким образом, данные действия могут служить инструментом определения эффективности образовательной среды (педагогической системы, образовательных технологий, материально-технических усмовий) относительно развития творческих способностей учащихся.

Собранный эмпирический материал (результаты анкетирования учащихся) обрабатывался с помощью компьютеризированной процедуры кластерного анализа. Нам удалось обнаружить развитие представлений учащихся о креативной мичности, которое сопровождается у учащихся рефмексивной оценкой собственного "Я"

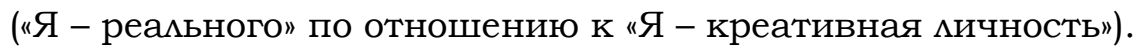

Оказалось, что в 10-х классах учащиеся представляют себя в качестве креативных субъектов только в будущем, когда они будут учиться в вузе и далее при осуществлении профессиональной деятельности. В их понимании креативный человек - это выдемяющаяся среди всех окружающих, яркая мичность, сочетающая в себе разцичные творческие способности.

Дия учащихся 11-х классов "Я - реальное" и "Я - креативная Аичность» очутились в одном кластере (группе семантически близких понятий). Это означает, что они в большей степени отождествцяют себя с креативной мичностью, чем 10-классники. Представление у выпус- 
кников о креативном человеке становится более насыщенным и конкретным. В качестве базовой характеристики они выделяют широкий интерес к познанию, считают, что именно он и мотивация стимулируют человека к действию и творческому развитию; отмечают также такие значимые качества креативной мичности, как толерантность к неопределенности, независимость взглядов, гибкость мышления.

Выяснилось, что среди приоритетных характеристик креативной среды общими и для 10-и, и 11-классников являются "возможность индивидуального самовыражения", "доступ к духовным благам", "обучение, нацеменное на развитие таланта". А такие характеристики, как "коммуникативная компетентность педагога" и "положительный эмоциональный фон", имеют более существенное значение для 10-классников, чем для 11-классников. Мы полагаем, что это связано с процессом адаптации 10-классников в начальный период обучения в гимназии, когда педагогическая и психологическая поддержка со стороны педагога особо важна дмя ученика.

В 11-х классах с помощью факторного анализа выделены, как и в 10-х классах, два фактора, которые характеризуют представления учащихся о креативной развивающей среде. Первый фактор - "среда, стимулирующая развитие индивидуальности", второй фактор - "вариативность развивающей среды". Несмотря на то, что многие характеристики креативной среды в сознании 10-и и 11-классников совпадают, наблюдается некоторое смещение приоритетов. Например, если в 10-х классах такая характеристика креативной среды, как "возможность стимулирующей деятельности", имеет очень высокую весовую нагрузку, то в 11-х классах эта характеристика вообще выпадает из ведущих факторов. Это говорит о том, что она стала неактуальной, ее значимость дмя старшеклассников в плане развития творческости в значительной степени утрачена. Можно предположить, что более значимой для 11-классников стала внутренняя мотивация творческого развития, а не внешняя. Такое смещение мы относим к положительному влиянию образовательной среды на мичностное развитие учащихся.

Во втором факторе "вариативность развивающей среды" несколько утратила свое значение такая характеристика среды, как "усмовия конкуренции", сместившись с первого рейтинга в 10-х классах на последний в 11-х, уступив позиции "разнообразию видов учебнопознавательной деятельности", "наличию креативного педагога", "от- 
сутствию препятствий самореализации" и др. Такое изменение мы также связываем с динамикой развития внутренней мотивации творческой деятельности, пониманием учащимися ценности внутренних (Аичностных) мотивов развития, а не внешних. Более значимой в представлении 11-классников стала характеристика среды "наличие времени на обдумывание». Такое изменение, с нашей точки зрения, является вполне закономерным, поскольку истинно творческий процесс нельзя регламентировать по времени и образовательная среда должна предусматривать время для выполнения творческих задач.

В заключение отметим, что сочетание количественных и качественных методов в исследовании (измерении, обработке, интерпретации результатов) дало нам возможность глубже понять и оценить взаимосвязь развития креативного потенциала ребенка и педагогической среды. Выявлено значимое влияние применения в педагогическом процессе вариативных, многопрофильных учебных программ, креативных методов организации учебно-познавательной деятельности, разцичных эвристических приемов, развивающих творческие способности учащихся и их представления о креативной мичности и творческой среде.

\section{Аитература}

1. Гапонцева М. Г., Федоров В. А., Гапонцев В. П. Понятия геометрии фракталов как язык объектов педагогики и теории научного знания // Образование и наука. Изв. УрО РАО. 2009. № 4 (61). C. 6-22.

2. Захарова И. Г. Модели и методы математической статистики в психолого-педагогическом исследовании // Опытно-экспериментальное исследование в педагогике: могика, содержание, инструментарий / под ред. А. Ф. Закировой. Тюмень: ТОГИРРО, 2012. 32 c.

3. Кочнев В. П. Проблемные математические задачи как средство развития творческих способностей учащихся // Образование и наука. Изв. УрО РАО. 2011. № 3 (82). С. 108-116.

4. Кэмпбелц Д. Модели экспериментов в социальной психологии и прикладных исследованиях. СПб.: Социально-психологический центр, 1996. 396 с.

5. Равен Д. Педагогическое тестирование. Проблемы, заблуждения, перспективы: пер. с англ. М.: Когито-Центр, 1999. 144 с. 